\title{
Fast convergence of generalized DeTemple sequences and the relation to the Riemann zeta function
}

\author{
Shanhe $\mathrm{Wu}^{1 *}$ and Gabriel Bercu
}

\section{"Correspondence:}

shanhewu@gmail.com

'Department of Mathematics,

Longyan University, Longyan, Fujian

364012, P.R. China

Full list of author information is

available at the end of the article

\begin{abstract}
In this paper, we introduce new sequences, which generalize the celebrated DeTemple sequence, having enhanced speed of convergence. We also give a new representation for Euler's constant in terms of the Riemann zeta function evaluated at positive odd integers.
\end{abstract}

MSC: 41A60; 41A25; 57Q55

Keywords: DeTemple sequence; convergence; Euler-Mascheroni constant; approximation; Riemann zeta function

\section{Introduction and preliminaries}

As is well known, the celebrated Euler-Mascheroni sequence,

$$
\gamma_{n}=1+\frac{1}{2}+\cdots+\frac{1}{n}-\log n \quad(n \geq 1)
$$

is convergent to the Euler-Mascheroni constant $\gamma=0.5772156649015328 \ldots$, and the rate of convergence is $n^{-1}$. In order to improve the rate of convergence, in [1], DeTemple modified the argument of the logarithm and showed that the sequence

$$
R_{n}=1+\frac{1}{2}+\cdots+\frac{1}{n}-\log \left(n+\frac{1}{2}\right)
$$

converges quadratically to $\gamma$. For more details as regards the approximation of the Euler constant with a very high accuracy, we mention the work of Sweeney [2], Bailey [3] and Alzer and Koumandos [4].

There are many famous unsolved problems associated with the properties of this constant; for example it is not known yet if the Euler constant is irrational. The Euler constant is an unusual constant and it seems unrelated to other known constants. However, there are many areas in which Euler's constant appears, for example, probability theory, random matrix theory and Riemann hypothesis, etc. Recently, Agarwal et al. [5], Wang et al. [6-9] and Yang et al. [10] showed that the Euler-Mascheroni constant has important applications in the field of special functions. In a sense, its appearance in different mathematical areas can be regarded as possible connections between these subjects.

(c) The Author(s) 2017. This article is distributed under the terms of the Creative Commons Attribution 4.0 International License (http://creativecommons.org/licenses/by/4.0/), which permits unrestricted use, distribution, and reproduction in any medium, provided you give appropriate credit to the original author(s) and the source, provide a link to the Creative Commons license, and indicate if changes were made. 
It is the aim of this article to develop a new direction to accelerate the speed of convergence of the sequence $\left(R_{n}\right)_{n \geq 1}$. For this purpose, we consider the sequence

$$
\omega_{n}=1+\frac{1}{2}+\cdots+\frac{1}{n}+x_{n}-\log \left(n+b_{n}\right),
$$

where $\left(x_{n}\right)_{n \geq 1}$ and $\left(b_{n}\right)_{n \geq 1}$ are suitable sequences, which are chosen in order to increase the speed of convergence of $\left(\omega_{n}\right)_{n \geq 1}$.

Note that we can write $\omega_{n}$ in the form of

$$
\omega_{n}=1+\frac{1}{2}+\cdots+\frac{1}{n}-\log n+x_{n}+\log \frac{1}{1+\frac{b_{n}}{n}} .
$$

If $\lim _{n \rightarrow \infty} x_{n}=x$, with $x \in \mathbb{R}$, and $\lim _{n \rightarrow \infty} \frac{b_{n}}{n}=0$, then the sequence $\left(\omega_{n}\right)_{n \geq 1}$ is convergent to $\gamma+x$.

To calculate the rate of convergence, we will utilize the Stolz lemma in the case of $\frac{0}{0}$.

Lemma 1.1 Let $\left(u_{n}\right)_{n \geq 1}$ and $\left(v_{n}\right)_{n \geq 1}$ be two sequences of real numbers, having the following properties:

(i) $\lim _{n \rightarrow \infty} u_{n}=\lim _{n \rightarrow \infty} v_{n}=0$;

(ii) the sequence $\left(v_{n}\right)_{n \geq 1}$ is strictly decreasing;

(iii) there exists $\lim _{n \rightarrow \infty} \frac{u_{n}-u_{n-1}}{v_{n}-v_{n-1}}=\ell$, with $\ell \in \mathbb{R}$.

Then the sequence $\left(\frac{u_{n}}{v_{n}}\right)_{n \geq 1}$ is convergent, and $\lim _{n \rightarrow \infty} \frac{u_{n}}{v_{n}}=\ell$.

In the following, we are concerned with finding non-vanishing limits of the form

$$
\ell=\lim _{n \rightarrow \infty} n^{k}\left(\omega_{n}-\omega\right)=\lim _{n \rightarrow \infty} \frac{\omega_{n}-\omega}{\frac{1}{n^{k}}}
$$

where $\omega=\lim _{n \rightarrow \infty} \omega_{n}$. The result will be better as the natural number $k$ is large.

Note that

$$
\lim _{n \rightarrow \infty} \frac{\frac{1}{n^{k+1}}}{\frac{1}{n^{k}}-\frac{1}{(n-1)^{k}}}=-\frac{1}{k}
$$

using Lemma 1.1, it is enough to find the following limit:

$$
\ell=\lim _{n \rightarrow \infty} \frac{\omega_{n}-\omega_{n-1}}{\frac{1}{n^{k}}-\frac{1}{(n-1)^{k}}}=-\frac{1}{k} \lim _{n \rightarrow \infty} n^{k+1}\left(\omega_{n}-\omega_{n-1}\right) .
$$

We shall write the difference $\omega_{n}-\omega_{n-1}$ as a power series of $n^{-1}$.

First, we arrange the difference $\omega_{n}-\omega_{n-1}$ as

$$
\begin{aligned}
\omega_{n}-\omega_{n-1} & =\frac{1}{n}+x_{n}-x_{n-1}-\log \left(n+b_{n}\right)+\log \left(n-1+b_{n-1}\right) \\
& =\frac{1}{n}+x_{n}-x_{n-1}-\log \left[n\left(1+\frac{b_{n}}{n}\right)\right]+\log \left[n\left(1+\frac{b_{n-1}-1}{n}\right)\right] \\
& =\frac{1}{n}+x_{n}-x_{n-1}-\log \left(1+\frac{b_{n}}{n}\right)+\log \left(1+\frac{b_{n-1}-1}{n}\right) .
\end{aligned}
$$


Then, using the power series representation of $\log (1+x)$, we obtain

$$
\begin{aligned}
\omega_{n}-\omega_{n-1}= & \frac{1}{n}+x_{n}-x_{n-1}-\left[\frac{b_{n}}{n}-\frac{1}{2}\left(\frac{b_{n}}{n}\right)^{2}+\frac{1}{3}\left(\frac{b_{n}}{n}\right)^{3}-\frac{1}{4}\left(\frac{b_{n}}{n}\right)^{4}+\cdots\right] \\
& +\left[\frac{b_{n-1}-1}{n}-\frac{1}{2}\left(\frac{b_{n-1}-1}{n}\right)^{2}+\frac{1}{3}\left(\frac{b_{n-1}-1}{n}\right)^{3}-\frac{1}{4}\left(\frac{b_{n-1}-1}{n}\right)^{4}+\cdots\right] .
\end{aligned}
$$

After some arrangement, we have

$$
\begin{aligned}
\omega_{n}-\omega_{n-1}= & \frac{1}{n}+x_{n}-x_{n-1}-\frac{b_{n}}{n}+\frac{b_{n-1}-1}{n}+\frac{b_{n}^{2}-\left(b_{n-1}-1\right)^{2}}{2 n^{2}}-\frac{b_{n}^{3}-\left(b_{n-1}-1\right)^{3}}{3 n^{3}} \\
& +\frac{b_{n}^{4}-\left(b_{n-1}-1\right)^{4}}{4 n^{4}}+O\left(\frac{1}{n^{5}}\right)
\end{aligned}
$$

or more convenient

$$
\begin{aligned}
\omega_{n}-\omega_{n-1}= & x_{n}-x_{n-1}-\frac{b_{n}-b_{n-1}}{n}+\frac{b_{n}^{2}-\left(b_{n-1}-1\right)^{2}}{2 n^{2}} \\
& -\frac{b_{n}^{3}-\left(b_{n-1}-1\right)^{3}}{3 n^{3}}+\frac{b_{n}^{4}-\left(b_{n-1}-1\right)^{4}}{4 n^{4}}+O\left(\frac{1}{n^{5}}\right) .
\end{aligned}
$$

The above relational expression (1.1) is useful, it will be used to establish our main results in subsequent sections.

\section{Sequences of DeTemple type and relevant results}

Let us consider certain special cases of $x_{n}-x_{n-1}$ and $b_{n}-b_{n-1}$, which will enable us to establish the generalizations of some sequences from DeTemple [1] and Mortici [11].

CASE 1. Suppose $x_{n}-x_{n-1}=0$, for all $n \geq 2$ and $b_{n}-b_{n-1}=0$, for all $n \geq 2$.

In this case the sequences $\left(x_{n}\right)_{n \geq 1}$ and $\left(b_{n}\right)_{n \geq 1}$ are constant; let $x_{n}=c$, for all $n \geq 1$ and $b_{n}=b$, for all $n \geq 1$.

In (1.1), the coefficient of $\frac{1}{2 n^{2}}$ becomes $b_{n}^{2}-\left(b_{n-1}-1\right)^{2}=b^{2}-(b-1)^{2}=2 b-1$.

SUBCASE 1.1. If $b=\frac{1}{2}$, then the coefficient of $\frac{1}{n^{2}}$ vanishes and

$$
\ell=\lim _{n \rightarrow \infty} n^{2}\left(\omega_{n}-\omega\right)=-\frac{1}{2} \lim _{n \rightarrow \infty} n^{3}\left[\frac{1}{12 n^{3}}+O\left(\frac{1}{n^{4}}\right)\right]=-\frac{1}{24}
$$

therefore the speed of convergence is $n^{-2}$.

Finally, we obtain the sequence

$$
\omega_{n}=1+\frac{1}{2}+\cdots+\frac{1}{n}+c-\log \left(n+\frac{1}{2}\right)
$$

which for $c=0$ is the celebrated DeTemple sequence [1].

SUBCASE 1.2. If $b \neq \frac{1}{2}$, then the term in $\frac{1}{n^{2}}$ is present, therefore the speed of convergence is $n^{-1}$.

CASE 2. If $b_{n}-b_{n-1}=0$, for all $n \geq 2$, then the sequence $\left(b_{n}\right)_{n \geq 1}$ is constant. Suppose $b_{n}=b$, for all $n \geq 1$ and $b \neq \frac{1}{2}$. Then

$$
\omega_{n}-\omega_{n-1}=x_{n}-x_{n-1}+\frac{2 b-1}{2 n^{2}}-\frac{3 b^{2}-3 b+1}{3 n^{3}}+O\left(\frac{1}{n^{4}}\right) .
$$


SubCASE 2.1. Suppose $x_{n}-x_{n-1}+\frac{2 b-1}{2 n^{2}}=0$ for all $n \geq 2$. Taking the sum, we obtain

$$
x_{n}=x_{1}+\frac{1-2 b}{2}\left(\frac{1}{2^{2}}+\frac{1}{3^{2}}+\cdots+\frac{1}{n^{2}}\right) \text {. }
$$

The sequence $\left(\omega_{n}\right)$ becomes

$$
\omega_{n}=1+\frac{1}{2}+\cdots+\frac{1}{n}+x_{1}+\frac{1-2 b}{2}\left(\frac{1}{2^{2}}+\frac{1}{3^{2}}+\cdots+\frac{1}{n^{2}}\right)-\log (n+b)
$$

or, in a more suitable form,

$$
\omega_{n}=1+x_{1}+\sum_{k=2}^{n} \frac{2 k+1-2 b}{2 k^{2}}-\log (n+b)
$$

The speed of convergence of $\left(\omega_{n}\right)_{n \geq 1}$ is $n^{-2}$. Note that the logarithm function in the above expression has definition for $b \geq 0$. Therefore, we obtain the following result.

Theorem 2.1 If we denote by $\left(\omega_{n}\right)_{n \geq 1}$ the sequence (2.1) and by $\omega$ its limit, then $\left(\omega_{n}\right)_{n \geq 1}$ converges quadratic to $\omega$.

For $b=0$, we obtain the sequence defined in Theorem 3.1 of [11].

SubCASE 2.2. Suppose

$$
x_{n}-x_{n-1}+\frac{2 b-1}{2 n^{2}}+\frac{-3 b^{2}+3 b-1}{3 n^{3}}=0 \quad \text { for all } n \geq 2 \text {. }
$$

Summing side by side, we obtain

$$
x_{n}=x_{1}+\sum_{k=2}^{n}\left(\frac{1-2 b}{2} \cdot \frac{1}{k^{2}}+\frac{3 b^{2}-3 b+1}{3} \cdot \frac{1}{k^{3}}\right) \text {. }
$$

The sequence $\left(\omega_{n}\right)$ has the form

$$
\omega_{n}=1+x_{1}+\sum_{k=2}^{n}\left(\frac{1}{k}+\frac{1-2 b}{2} \cdot \frac{1}{k^{2}}+\frac{3 b^{2}-3 b+1}{3} \cdot \frac{1}{k^{3}}\right)-\log (n+b) .
$$

The speed of convergence is $n^{-3}$. Note that the $\operatorname{logarithm} \log (n+b)$ has definition for $b \geq 0$. This leads to the following assertion.

Theorem 2.2 If we denote by $\left(\omega_{n}\right)_{n \geq 1}$ the sequence (2.2) and by $\omega$ its limit, then $\left(\omega_{n}\right)_{n \geq 1}$ converges cubic to $\omega$.

For $b=0$, we obtain the sequence defined in Theorem 3.2 of [11].

\section{Generalized DeTemple sequences}

In this section we shall establish two generalized DeTemple sequences in terms of the condition $b_{n}+b_{n-1}-1=0$. 


\section{CASE 1. Suppose}

$$
x_{n}-x_{n-1}-\frac{b_{n}-b_{n-1}}{n}+\frac{b_{n}^{2}-\left(b_{n-1}-1\right)^{2}}{2 n^{2}}=0 \quad(n \geq 2) .
$$

We would like to find the sequence $\left(b_{n}\right)_{n \geq 1}$ such that $b_{n}+b_{n-1}-1=0$ (this vanishes; the nominator $\left.b_{n}^{2}-\left(b_{n-1}-1\right)^{2}=0\right)$. It follows that $b_{n}=-b_{n-1}+1$. Therefore $b_{n}=\frac{(-1)^{n-1} \cdot\left(2 b_{1}-1\right)+1}{2}$, and this satisfies the initial condition $\lim _{n \rightarrow \infty} \frac{b_{n}}{n}=0$. On the other hand, one has $b_{n}-b_{n-1}=$ $(-1)^{n-1}\left(2 b_{1}-1\right)$.

Equation (3.1) becomes

$$
x_{n}-x_{n-1}=\frac{(-1)^{n-1}}{n}\left(2 b_{1}-1\right) .
$$

Taking the sum from 2 to $n$ and fixing $x_{1}=2 b_{1}-1$, we obtain

$$
x_{n}=\left(2 b_{1}-1\right)\left(\frac{1}{1}-\frac{1}{2}+\frac{1}{3}-\frac{1}{4}+\cdots+\frac{(-1)^{n-1}}{n}\right) \text {. }
$$

We remark that $\left(x_{n}\right)_{n \geq 1}$ is the Leibniz's sequence multiplied by the constant $\left(2 b_{1}-1\right)$. This sequence is convergent, having the limit

$$
\lim _{n \rightarrow \infty} x_{n}=\left(2 b_{1}-1\right) \log 2 .
$$

Therefore, we obtain the sequence

$$
\begin{aligned}
\omega_{n}= & +\frac{1}{2}+\cdots+\frac{1}{n}+\left(2 b_{1}-1\right)\left(1-\frac{1}{2}+\frac{1}{3}-\frac{1}{4}+\cdots+\frac{(-1)^{n-1}}{n}\right) \\
& -\log \left[n+\frac{(-1)^{n-1} \cdot\left(2 b_{1}-1\right)+1}{2}\right]
\end{aligned}
$$

or, in more suitable form,

$$
\omega_{n}=\sum_{k=1}^{n} \frac{1+\left(2 b_{1}-1\right)(-1)^{k-1}}{k}-\log \left[n+\frac{(-1)^{n-1} \cdot\left(2 b_{1}-1\right)+1}{2}\right],
$$

with speed of convergence $n^{-2}$ and limit $\gamma+\left(2 b_{1}-1\right) \log 2$.

Note that the logarithm function in the above expression has definition for $b_{1} \in(0,1)$. Hence, we have the following result.

Theorem 3.1 If we denote by $\left(w_{n}\right)_{n \geq 1}$ the sequence (3.2), then $\left(w_{n}\right)_{n \geq 1}$ converges quadratically to its limit $\gamma+\left(2 b_{1}-1\right) \log 2$.

It is clear that for $b_{1}=\frac{1}{2}$ we obtain DeTemple sequence.

CASE 2. Note that

$$
\begin{aligned}
\omega_{n}-\omega_{n-1}= & x_{n}-x_{n-1}-\frac{b_{n}-b_{n-1}}{n}-\frac{b_{n}^{3}-\left(b_{n-1}-1\right)^{3}}{3 n^{3}} \\
& -\cdots-\frac{b_{n}^{2 p+1}-\left(b_{n-1}-1\right)^{2 p+1}}{(2 p+1) n^{2 p+1}}+O\left(\frac{1}{n^{2 p+3}}\right) .
\end{aligned}
$$


As above, we consider the sequence $b_{n}=\frac{(-1)^{n-1}\left(2 b_{1}-1\right)+1}{2}, b_{1} \in(0,1)$.

We have seen that it satisfies the equality $b_{n}+b_{n-1}-1=0$. Then $b_{n}^{2}-\left(b_{n-1}-1\right)^{2}=0$ and, in general,

$$
b_{n}^{2 k}-\left(b_{n-1}-1\right)^{2 k}=0, \quad k=1,2, \ldots
$$

Suppose

$$
x_{n}-x_{n-1}=\frac{b_{n}-b_{n-1}}{n}+\frac{b_{n}^{3}-\left(b_{n-1}-1\right)^{3}}{3 n^{3}}+\cdots+\frac{b_{n}^{2 p+1}-\left(b_{n-1}-1\right)^{2 p+1}}{(2 p+1) n^{2 p+1}} \text {. }
$$

From $b_{n}+b_{n-1}-1=0$, one has $b_{n-1}-1=-b_{n}$. Further, it follows that

$$
b_{n}^{2 k+1}-\left(b_{n-1}-1\right)^{2 k+1}=b_{n}^{2 k+1}-\left(-b_{n}\right)^{2 k+1}=2 b_{n}^{2 k+1} .
$$

On the other hand,

$$
\begin{aligned}
\frac{b_{n}-b_{n-1}}{n} & =\frac{b_{1}(-1)^{n-1}+\frac{1-(-1)^{n-1}}{2}-b_{1}(-1)^{n-2}-\frac{1-(-1)^{n-2}}{2}}{n} \\
& =\frac{(-1)^{n-1}\left(2 b_{1}-1\right)}{n} .
\end{aligned}
$$

Hence, we deduce that

$$
x_{n}-x_{n-1}=\frac{(-1)^{n-1}\left(2 b_{1}-1\right)}{n}+\frac{2 b_{n}^{3}}{3 n^{3}}+\frac{2 b_{n}^{5}}{5 n^{5}}+\cdots+\frac{2 b_{n}^{2 p+1}}{(2 p+1) n^{2 p+1}} .
$$

Taking the sum for $k$ from 2 to $n$, we have

$$
x_{n}-x_{1}=\sum_{k=2}^{n}\left[\frac{(-1)^{k-1}\left(2 b_{1}-1\right)}{k}+\frac{2 b_{k}^{3}}{3 k^{3}}+\cdots+\frac{2 b_{k}^{2 p+1}}{(2 p+1) k^{2 p+1}}\right] \text {. }
$$

If we fix

$$
x_{1}=2 b_{1}-1+\frac{2 b_{1}^{3}}{3}+\frac{2 b_{1}^{5}}{5}+\cdots+\frac{2 b_{1}^{2 p+1}}{2 p+1}
$$

then we obtain

$$
x_{n}=\sum_{k=1}^{n}\left[\frac{(-1)^{k-1}\left(2 b_{1}-1\right)}{k}+\frac{2 b_{k}^{3}}{3 k^{3}}+\cdots+\frac{2 b_{k}^{2 p+1}}{(2 p+1) k^{2 p+1}}\right] \text {. }
$$

Hence,

$$
\begin{aligned}
\omega_{n}= & +\frac{1}{2}+\cdots+\frac{1}{n}+\sum_{k=1}^{n}\left[\frac{(-1)^{k-1}\left(2 b_{1}-1\right)}{k}+\frac{2 b_{k}^{3}}{3 k^{3}}+\cdots+\frac{2 b_{k}^{2 p+1}}{(2 p+1) k^{2 p+1}}\right] \\
& -\log \left[n+\frac{(-1)^{n-1}\left(2 b_{1}-1\right)+1}{2}\right]
\end{aligned}
$$




$$
\begin{aligned}
= & \sum_{k=1}^{n} \frac{1}{k}+\sum_{k=1}^{n}\left[\frac{(-1)^{k-1}\left(2 b_{1}-1\right)}{k}+\frac{2 b_{k}^{3}}{3 k^{3}}+\cdots+\frac{2 b_{k}^{2 p+1}}{(2 p+1) k^{2 p+1}}\right] \\
& -\log \left[n+\frac{(-1)^{n-1}\left(2 b_{1}-1\right)+1}{2}\right] \\
= & \sum_{k=1}^{n}\left[2 \cdot \frac{(-1)^{k-1}\left(2 b_{1}-1\right)+1}{2 k}+\frac{2}{3}\left(\frac{(-1)^{k-1}\left(2 b_{1}-1\right)+1}{2 k}\right)^{3}\right. \\
& \left.+\cdots+\frac{2}{2 p+1}\left(\frac{(-1)^{k-1}\left(2 b_{1}-1\right)+1}{2 k}\right)^{2 p+1}\right] \\
& -\log \left[n+\frac{(-1)^{n-1}\left(2 b_{1}-1\right)+1}{2}\right] .
\end{aligned}
$$

Putting $a_{k}=\frac{(-1)^{k-1}\left(2 b_{1}-1\right)+1}{2 k}$, then $\lim _{k \rightarrow \infty} a_{k}=0$.

Further,

$$
\omega_{n}=2 \sum_{k=1}^{n}\left(\frac{a_{k}}{1}+\frac{a_{k}^{3}}{3}+\cdots+\frac{a_{k}^{2 p+1}}{2 p+1}\right)-\log \left(n+n a_{n}\right) .
$$

Consequently, we get the following result.

\section{Theorem 3.2 The sequence}

$$
\omega_{n}=2 \sum_{k=1}^{n}\left(\frac{a_{k}}{1}+\frac{a_{k}^{3}}{3}+\cdots+\frac{a_{k}^{2 p+1}}{2 p+1}\right)-\log \left(n+n a_{n}\right)
$$

has speed of convergence $n^{-2 p-2}$, where $a_{k}=\frac{(-1)^{k-1}\left(2 b_{1}-1\right)+1}{2 k}, b_{1} \in(0,1)$.

It is easy to observe that $a_{2 k}=\frac{1-b_{1}}{2 k}$ and $a_{2 k+1}=\frac{b_{1}}{2 k+1}$.

In order to find the limit of the sequence $\left(\omega_{n}\right)_{n \geq 1}$, we note that it is related to the harmonic sequence $\zeta_{n}(s)=\sum_{k=1}^{n} \frac{1}{k^{s}}$.

For $s>1$, the limit of this sequence defined the celebrated Riemann zeta function $\zeta(s)$, which is very important in mathematics. The speed of convergence of this sequence to its limit is described by the double inequality

$$
\frac{1}{(s-1)(n+1)^{s-1}}<\zeta(s)-\zeta_{n}(s)<\frac{1}{(s-1) n^{s-1}} .
$$

Thus the sequence $\left(\zeta_{n}(s)\right)_{n \geq 1}$ converges with speed of convergence $n^{1-s}$.

A direct calculation gives

$$
\begin{aligned}
\sum_{k=1}^{\infty} \frac{2 a_{k}^{2 s+1}}{2 s+1} & =\frac{2}{2 s+1}\left(a_{1}^{2 s+1}+a_{2}^{2 s+1}+\cdots+a_{n}^{2 s+1}+\cdots\right) \\
& =\frac{2}{2 s+1}\left[\left(\frac{b_{1}}{1}\right)^{2 s+1}+\left(\frac{1-b_{1}}{2}\right)^{2 s+1}+\left(\frac{b_{1}}{3}\right)^{2 s+1}+\left(\frac{1-b_{1}}{4}\right)^{2 s+1}+\cdots\right] \\
& =\frac{2}{2 s+1}\left[b_{1}^{2 s+1} \cdot\left(\frac{1}{1^{2 s+1}}+\frac{1}{3^{2 s+1}}+\frac{1}{5^{2 s+1}}+\cdots\right)\right.
\end{aligned}
$$




$$
\begin{aligned}
& \left.+\left(1-b_{1}\right)^{2 s+1} \cdot\left(\frac{1}{2^{2 s+1}}+\frac{1}{4^{2 s+1}}+\frac{1}{6^{2 s+1}}+\cdots\right)\right] \\
= & \frac{2}{2 s+1}\left[b_{1}^{2 s+1}\left(\frac{1}{1^{2 s+1}}+\frac{1}{2^{2 s+1}}+\frac{1}{3^{2 s+1}}+\frac{1}{4^{2 s+1}}+\frac{1}{5^{2 s+1}}+\cdots\right)\right. \\
& -b_{1}^{2 s+1}\left(\frac{1}{2^{2 s+1}}+\frac{1}{4^{2 s+1}}+\frac{1}{6^{2 s+1}}+\cdots\right) \\
& \left.+\left(1-b_{1}\right)^{2 s+1} \cdot \frac{1}{2^{2 s+1}}\left(\frac{1}{1^{2 s+1}}+\frac{1}{2^{2 s+1}}+\frac{1}{3^{2 s+1}}+\cdots\right)\right] .
\end{aligned}
$$

Hence,

$$
\begin{aligned}
\sum_{k=1}^{\infty} \frac{2 a_{k}^{2 s+1}}{2 s+1}= & \frac{2}{2 s+1}\left[b_{1}^{2 s+1} \cdot \zeta(2 s+1)-b_{1}^{2 s+1} \cdot \frac{1}{2^{2 s+1}} \zeta(2 s+1)\right. \\
& \left.+\left(1-b_{1}\right)^{2 s+1} \cdot \frac{1}{2^{2 s+1}} \zeta(2 s+1)\right] \\
= & \frac{2}{2 s+1} \cdot \frac{b_{1}^{2 s+1}\left(2^{2 s+1}-1\right)+\left(1-b_{1}\right)^{2 s+1}}{2^{2 s+1}} \cdot \zeta(2 s+1) \\
= & \frac{b_{1}^{2 s+1}\left(2^{2 s+1}-1\right)+\left(1-b_{1}\right)^{2 s+1}}{(2 s+1) \cdot 2^{2 s}} \cdot \zeta(2 s+1),
\end{aligned}
$$

where $s \in\{1,2, \ldots, p\}$.

We have showed in Case 1 that $\lim _{n \rightarrow \infty} \sum_{k=1}^{n} 2 a_{k}-\log \left(n+n a_{n}\right)=\gamma+\left(2 b_{1}-1\right) \log 2$.

Therefore, we obtain the following theorem.

Theorem 3.3 The sequence $\left(\omega_{n}\right)_{n \geq 1}$ defined by Theorem 3.2 converges to

$$
\gamma+\left(2 b_{1}-1\right) \log 2+\sum_{s=1}^{p} \frac{b_{1}^{2 s+1}\left(2^{2 s+1}-1\right)+\left(1-b_{1}\right)^{2 s+1}}{2^{2 s}(2 s+1)} \zeta(2 s+1)
$$

with speed of convergence $n^{-2 p-2}$.

If we take $b_{1}=\frac{1}{2}$ in Theorem 3.3, then $a_{k}=\frac{1}{2 k}$,

$$
\begin{aligned}
\omega_{n}= & \sum_{k=1}^{n} 2\left[\frac{1}{2 k}+\frac{1}{3 \cdot 2^{3} k^{3}}+\cdots+\frac{1}{(2 p+1) 2^{2 p+1} k^{2 p+1}}\right] \\
& -\log \left(n+\frac{1}{2}\right) \\
= & R_{n}+\sum_{k=1}^{n}\left[\frac{1}{3 \cdot 2^{2} k^{3}}+\cdots+\frac{1}{(2 p+1) 2^{2 p} k^{2 p+1}}\right],
\end{aligned}
$$

where

$$
R_{n}=1+\frac{1}{2}+\cdots+\frac{1}{n}-\log \left(n+\frac{1}{2}\right)
$$

Thus, we obtain the following assertion. 
Corollary 3.1 The sequence $\left(\omega_{n}\right)_{n \geq 1}$ defined by (3.3) converges to

$$
\gamma+\sum_{s=1}^{p} \frac{1}{(2 s+1) 2^{2 s}} \zeta(2 s+1)
$$

with speed of convergence $n^{-2 p-2}$.

Particular Cases. For $p=0$, the sequence $\left(\omega_{n}\right)_{n \geq 1}$ is a DeTemple sequence.

For $p=1$, the sequence $\left(\omega_{n}\right)_{n \geq 1}$ is the sequence defined in Theorem 4.1 of [12].

For $p=2$, the sequence $\left(\omega_{n}\right)_{n \geq 1}$ is the sequence defined in Theorem 4.2 of [12].

We remark that the expressions from Theorem 3.3 and Corollary 3.1 concern the expansion of Euler's constant in terms of the Riemann zeta function evaluated at positive odd integers. Therefore the constant $\gamma$ can be approximated with a very high speed of convergence by the above expansions. For more details of the series representations of the Euler constant, we mention the work of Alzer, Karayannakis and Srivastava [12], Alzer and Koumandos [13] and Sofo [14].

\section{Conclusion}

Our method for accelerating the convergence of DeTemple type sequences is more general than the method introduced in [11] and [15] by Mortici. We support this statement by the large degree of freedom in choosing the sequences $\left(x_{n}\right)_{n \geq 1}$, and $\left(b_{n}\right)_{n \geq 1}$. In this way, we generalized sequences from [11] and introduced two new sequences which generalized DeTemple sequence $\left(R_{n}\right)_{n \geq 1}$ having faster convergence rate.

\section{Acknowledgements}

The work of the first author is supported by the Natural Science Foundation of Fujian Province of China under Grant 2016J01023 and the Foundation of Science and Technology Innovation Team of Longyan University under Grant 201503.

\section{Competing interests}

The authors declare that they have no competing interests.

Authors' contributions

All authors contributed equally and significantly in this paper. All authors read and approved the final manuscript.

\section{Author details}

'Department of Mathematics, Longyan University, Longyan, Fujian 364012, P.R. China. ${ }^{2}$ Department of Mathematics and Computer Sciences, "Dunărea de Jos" University of Galaţi, 111 Domnească Street, Galaţi, 800201, Romania.

\section{Publisher's Note}

Springer Nature remains neutral with regard to jurisdictional claims in published maps and institutional affiliations.

Received: 14 February 2017 Accepted: 27 April 2017 Published online: 12 May 2017

\section{References}

1. DeTemple, DW: A quicker convergence to Euler's constant. Am. Math. Mon. 100(5), 468-470 (1993)

2. Sweeney, DW: On the computation of Euler's constant. Math. Comput. 17, 170-178 (1963)

3. Bailey, DH: Numerical results on the transcendence of constants involving $\pi, e$ and Euler's constant. Math. Comput. 50, 275-281 (1988)

4. Alzer, H, Koumandos, S: Series representations for $\gamma$ and other mathematical constants. Anal. Math. 34(1), 1-8 (2008)

5. Agarwal, $\mathrm{P}, \mathrm{Chand}, \mathrm{M}$, Onur Kiymaz, I, Çetinkaya, A: A certain sequence of functions involving the Aleph function. Open Phys. 14(1), 187-191 (2016)

6. Wang, MK, Chu, YM: Refinements of transformation inequalities for zero-balanced hypergeometric functions. Acta Math. Sci. Ser. B Engl. Ed. 37(3), 607-622 (2017)

7. Wang, MK, Li, YM, Chu, YM: Inequalities and infinite product formula for Ramanujan generalized modular equation function. Ramanujan J. (2017). doi:10.1007/s11139-017-9888-3

8. Wang, MK, Chu, YM, Qiu, SL: Sharp bounds for generalized elliptic integrals of the first kind. J. Math. Anal. Appl. 429(2), 744-757 (2015) 
9. Wang, MK, Chu, YM, Song, YQ: Asymptotical formulas for Gaussian and generalized hypergeometric functions. Appl. Math. Comput. 276, 44-60 (2016)

10. Yang, ZH, Chu, YM, Zhang, XH: Sharp bounds for psi function. Appl. Math. Comput. 268, 1055-1063 (2015)

11. Mortici, C: On some Euler-Mascheroni type sequences. Comput. Math. Appl. 60, 2009-2014 (2010)

12. Alzer, H, Karayannakis, D, Srivastava, H: Series representations for some mathematical constants. J. Math. Anal. Appl. 320(1), 145-162 (2006)

13. Alzer, $\mathrm{H}$, Koumandos, S: Series and product representations for some mathematical constants. Period. Math. Hung. 58(1), 71-82 (2009)

14. Sofo, A: Harmonic nummbers of order two. Miskolc Math. Notes 13(2), 499-514 (2012)

15. Mortici, C: Fast convergences towards Euler-Mascheroni constant. Comput. Appl. Math. 29(3), 479-491 (2010)

Submit your manuscript to a SpringerOpen ${ }^{\odot}$ journal and benefit from:

- Convenient online submission

Rigorous peer review

- Immediate publication on acceptance

- Open access: articles freely available online

- High visibility within the field

- Retaining the copyright to your article 